\title{
ESTIMATION OF EXHAUST MANIFOLD PRESSURE IN TURBOCHARGED GASOLINE ENGINES WITH VARIABLE VALVE TIMING
}

\author{
Julia H. Buckland \\ Mrdjan Jankovic \\ Research and Advanced Engineering \\ Ford Motor Company \\ Dearborn, Michigan 48124 \\ Email: jbucklan@ford.com
}

\author{
J. W. Grizzle \\ J. S. Freudenberg \\ Dept. of Electrical Engineering and Computer Science \\ University of Michigan \\ Ann Arbor, Michigan 48109
}

\begin{abstract}
Feedforward A/F control in turbocharged gasoline engines with variable valve timing requires knowledge of exhaust manifold pressure, $P_{e}$. Physical conditions in the manifold make measurement costly, compelling manufacturers to implement some form of on-line estimation. Processor limitations and the calibration process, however, put constraints on estimator complexity. This paper assesses the feasibility of estimating $P_{e}$ with an algorithm that is computationally efficient and relatively simple to calibrate. A traditional reduced order linear observer is found to perform well but has too many calibration parameters for practical implementation. Using the performance of the observer as a benchmark, static estimation is explored by parameterizing the equilibrium values of $P_{e}$ with both the inputs and the outputs of the system. This nonlinear static estimate, combined with simple lead compensation, yields a practical observer implementation.
\end{abstract}

\section{INTRODUCTION}

Modern automotive emission control systems for gasoline engines rely heavily on feedforward air-fuel ratio $(\mathrm{A} / \mathrm{F})$ control to meet strict emissions regulations. For turbocharged applications, it has been shown [1] that knowledge of exhaust manifold pressure $\left(P_{e}\right)$ is helpful in meeting the stringent accuracy requirements of the feedforward controller. When variable valve timing is added, the significance of $P_{e}$ rises dramatically, since considerable valve overlap may occur over a large portion of the operating envelope. Measuring $P_{e}$ can be quite problematic, however, due to the harsh environment in the manifold. System cost and durability considerations drive a strong desire by automakers for an estimate of $P_{e}$ based on commonly measured signals.

Most turbocharged gasoline applications employ turbines equipped with wastegates that open to divert flow around the turbine to control boost. Both the turbine and an open wastegate can be considered flow restrictions in the exhaust path of the engine. This interpretation of the physical system implies that the pressure in the exhaust manifold varies with mass flow rate and the effective size of the restrictions.

The total effective restriction varies greatly with wastegate opening. For a given command from the powertrain control module (PCM), the wastegate position may vary from its minimum to maximum deflection depending on the operating condition. This position is not typically measured. Therefore the size of the flow restriction in the exhaust path is not accurately known, making estimation of exhaust manifold pressure quite difficult. This is in contrast to modern diesel applications which employ variable geometry turbines where vane position is readily available.

In addition, cost and durability concerns also discourage measurement of temperature or other useful properties downstream of the engine. Thus, $P_{e}$ must be inferred from measurements of signals significantly distant and often related through dynamic elements.

Nonetheless, cost and time to market pressures dictate a simple and efficient estimation algorithm. Online computing resources are limited in automotive-quality processors, while growing hardware complexity and regulatory requirements continue to increase the number of computations and memory requirements of the software. Not coincidentally, calibration effort is also increasing, with each additional task lengthening development time and increasing cost. As such, strategies are highly 
scrutinized prior to implementation and those with added states or complex calibration procedures must demonstrate a clear and significant advantage to gain acceptance.

Here we use model-based analysis to determine the feasibility of estimating $P_{e}$ with a simple, efficient algorithm that can be easily calibrated. First we consider a traditional reduced order linear observer. Although nonlinearities in the system lead to a large number of calibration parameters, this approach establishes feasibility of a solution and provides a benchmark for comparison. Static estimation is then explored. Analysis of a linearized, steady state model of the system leads to a static linear estimate of exhaust manifold pressure based on conditions in the intake. This estimator provides excellent accuracy when applied to the nonlinear model in steady state. Transient performance, however, is shown to be poor due to the slow dynamics connecting the intake and exhaust. Simple lead compensation of the nonlinear static estimate produces a practical implementation with excellent steady state accuracy and improved transient performance.

\section{SYSTEM DESCRIPTION}

The system under consideration is an I-3 turbocharged engine equipped with variable intake cam timing, a conventional pneumatically operated wastegate to control boost and an intercooler to increase charge density and reduce tendency for engine knock. A schematic of the system is shown in Figure 1.

The model used for concept development is described in [2]. This model includes most of the major components of the desired system, with a notable exception being the pneumatically actuated wastegate. The effects of wastegate are incorporated in the model through a virtual actuator, wastegate flow rate, by assuming flow through the valve is a known input. This enables investigation of the estimation problem, for this and future applications, without the limitations imposed by current actuator technology. by

A four state representation of the modeled system is given

$$
\begin{aligned}
\dot{x} & =f(x, u) \\
x & =\left[P_{i}, P_{t i p}, P_{e}, N_{t c}\right]^{T} \\
u & =\left[E T C, V C T, W_{w g}, N\right]^{T} \\
y & =\left[P_{i}, P_{t i p}\right]^{T}
\end{aligned}
$$

where $P_{i}$ is intake manifold pressure, $P_{t i p}$ is throttle inlet pressure, $P_{e}$ is exhaust manifold pressure, $N_{t c}$ is turbocharger shaft speed, ETC is throttle angle, $V C T$ is variable cam timing, $W_{w g}$ is flow rate through the wastegate and $N$ is engine speed.

Since engine speed is measured, this model representation uses $N$ as an input; and since temperature changes slowly compared to the remaining states, manifold temperature dynamics are ignored. This simplified representation of the turbocharged system facilitates formal analysis of the estimation problem.

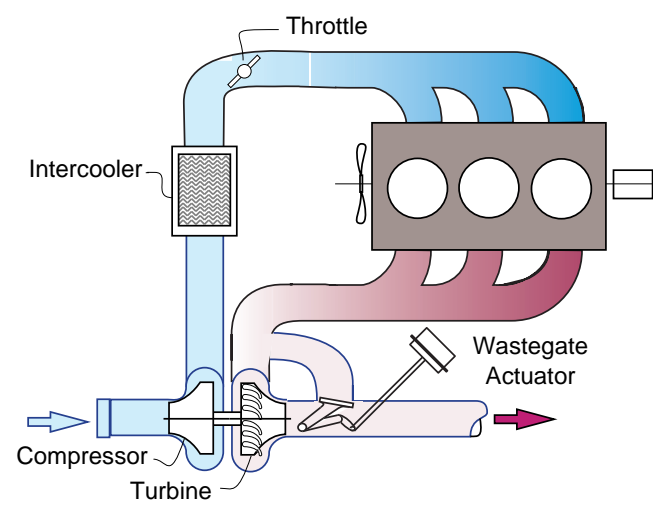

Figure 1. SYSTEM SCHEMATIC.

\section{OBSERVER DEVELOPMENT}

The system (1) has four states, two of which are measured, $P_{i}$ and $P_{t i p}$. Therefore, a reduced order linear observer is explored for estimation of $P_{e}$.

Consider a linear representation of (1) given by

$$
\begin{aligned}
& \delta \dot{x}=A \delta x+B \delta u \\
& \delta y=C \delta x
\end{aligned}
$$

where $\delta$ indicates deviation from the equilibrium point about which the system was linearized. In order to compare the relative influence of system parameters, (2) represents a system scaled relative to this equilibrium point such that deviation is defined in terms of fractional change. ${ }^{1}$

Following the development described in [3], the linear system is partitioned by grouping measured and unmeasured states as follows

$$
\begin{aligned}
& x_{1}=\left[P_{i}, P_{t i p}\right]^{T} \\
& x_{2}=\left[P_{e}, N_{t c}\right]^{T},
\end{aligned}
$$

such that

$$
\begin{aligned}
& \delta \dot{x}=\left[\begin{array}{l}
\delta \dot{x}_{1} \\
\delta \dot{x}_{2}
\end{array}\right]=\left[\begin{array}{ll}
A_{11} & A_{12} \\
A_{21} & A_{22}
\end{array}\right]\left[\begin{array}{l}
\delta x_{1} \\
\delta x_{2}
\end{array}\right]+\left[\begin{array}{l}
B_{1} \\
B_{2}
\end{array}\right] \delta u \\
& \delta y=\delta x_{1}=\left[\begin{array}{ll}
I_{2 x 2} & 0_{2 x 2}
\end{array}\right]\left[\begin{array}{l}
\delta x_{1} \\
\delta x_{2}
\end{array}\right] .
\end{aligned}
$$

The estimate $\hat{x}_{2}$ is defined by

$$
\begin{aligned}
\dot{z} & =A_{r} z+B_{r} w \\
\hat{x}_{2} & =C_{r} z+D_{r} w
\end{aligned}
$$

\footnotetext{
${ }^{1}$ For example, when $\delta W_{w g}=0.1$ wastegate flow is perturbed by $+10 \%$ from the equilibrium value for $W_{w g}$ used for linearization.
} 

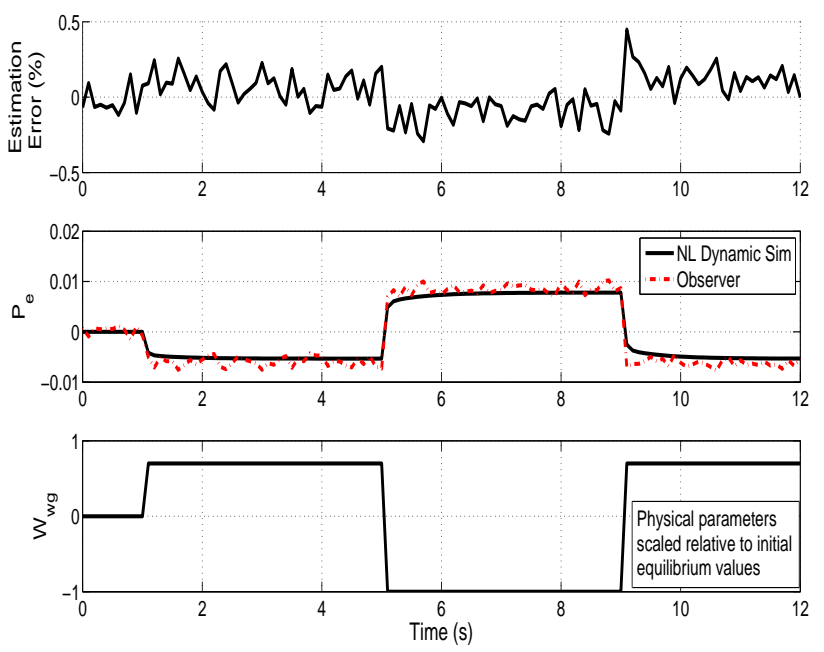

Figure 2. TRANSIENT PREDICTION OF $P_{e}$ USING A TRADITIONAL REDUCED ORDER OBSERVER.

where

$$
\begin{aligned}
w & =\left[\begin{array}{ll}
u & y
\end{array}\right]^{T} \\
A_{r} & =\left[\begin{array}{ll}
A_{22}-L A_{12}
\end{array}\right] \\
B_{r} & =\left[\begin{array}{ll}
\left(B_{2}-L B_{1}\right) & \left(A_{22}-L A_{12}\right) L+\left(A_{21}-L A_{11}\right)
\end{array}\right] \\
C_{r} & =\left[\begin{array}{ll}
I_{2 \times 2}
\end{array}\right] \\
D_{r} & =\left[\begin{array}{ll}
0_{2 \times 2} L &
\end{array} .\right.
\end{aligned}
$$

It can be shown that since $(A, C)$ is an observable pair, $\left(A_{22}, A_{12}\right)$ is an observable pair. It can also be shown that the estimation error, $\left(x_{2}-\hat{x}_{2}\right)$, goes to zero when the observer gain matrix $L$ is chosen such that $\left(A_{22}-L A_{12}\right)$ is asymptotically stable. For this application $L$ is chosen such that the eigenvalues of $\left(A_{22}-L A_{12}\right)$ are faster than the eigenvalues of $A_{22}$.

Observer performance is evaluated using a nonlinear simulation of the model described in [2]. The simulation model is modified by adding measurement noise with a maximum magnitude of approximately $\pm 1 \mathrm{kPa}$ to both $P_{i}$ and $P_{\text {tip }}$. These noisy raw measurement signals are then filtered at $30 \mathrm{rad} / \mathrm{sec}$ to represent the effects of digital signal processing typically employed by a PCM.

Simulation results showing the transient response of the observer to step changes in wastegate are shown by the dot-dashed line in Figure 2. ${ }^{2}$ This is compared with the simulated $P_{e}$ signal, represented by the solid line in the figure. The observer performs quite well, with relative error ${ }^{3}$ of less than $0.53 \%$ occuring during transient overshoots. This error is likely due to nonlinearities

\footnotetext{
${ }^{2}$ Physical parameters are scaled such that the figures show fractional change from the initial equilibrium value, for example, $P=\frac{P_{a}-P_{0}}{P_{a}}$ where $P_{a}$ is the actual physical parameter prior to scaling and $P_{0}$ is the equilibrium value at the start of simulation.

${ }^{3}$ Relative error is defined as $100\left(P_{e}-\hat{P}_{e}\right) / P_{e}$.
}

in the system that are not captured by the linear model.

\section{ESTIMATOR DEVELOPMENT}

The reduced order observer is quite effective, but it has two states and twenty parameters that must be calibrated, ${ }^{4}$ many of which must be scheduled with operating condition for this nonlinear system. Given the processor and calibration complexity limitations discussed in the Introduction, a simpler approach is preferred. Therefore we pursue a static representation of exhaust manifold pressure.

\section{Equilibrium Analysis}

Consider a continously differentiable nonlinear system

$$
\begin{aligned}
\dot{\xi} & =g(\xi, v) \\
\psi & =h(\xi)
\end{aligned}
$$

where $\xi \in \Re^{n}$ denotes the states of the nonlinear system, $v \in \Re^{p}$ represents the control inputs and the system outputs are given by $\psi \in \mathfrak{R}^{q}$. It's equilibrium points are given by the solutions of [4]

$$
\begin{aligned}
g(\xi, v) & =0 \\
\psi & =h(\xi) .
\end{aligned}
$$

Let $g\left(\xi_{0}, v_{0}\right)=0$ and $\psi=h\left(\xi_{0}\right)$ be one such solution and let

$$
\begin{aligned}
0 & =F \delta \xi+G \delta v \\
\delta \psi & =H \delta \xi,
\end{aligned}
$$

be the linearization of (3) about the equilibrium point. By the Implicit Function Theorem [5], there exists a continously differentiable function $\kappa(v, \psi)$, defined in a neighborhood of the equilibrium point, such that

$$
\begin{aligned}
0 & =g(\kappa(v, \psi), v) \\
\psi & =h(\kappa(v, \psi))
\end{aligned}
$$

as long as $\operatorname{rank}\left[\begin{array}{l}F \\ H\end{array}\right]=n$.

Such a function $\kappa$ is not (locally) unique when $n<p+q$. One way to fix a choice of $\kappa$ is to use only $n$ of the $p+q$ components of $v$ and $\psi$, or some combination thereof. To understand this more clearly rearrange (4) as follows

$$
\left[\begin{array}{l}
F \\
H
\end{array}\right] \delta \xi+\left[\begin{array}{cc}
G & 0 \\
0 & -I
\end{array}\right]\left[\begin{array}{c}
\delta v \\
\delta \psi
\end{array}\right]=0
$$

\footnotetext{
${ }^{4}$ Each matrix element is a calibration parameter.
} 
This gives a set of overdetermined equations from which we can form an estimate of $\delta \xi$ or equivalently $\kappa(v, \psi)$.

With more equations than unknowns, we introduce a weighting matrix to allow us to place emphasis on measurements and states in which we have high confidence, while effectively disregarding those for which we do not. Specifically,

$$
Q\left[\begin{array}{l}
F \\
H
\end{array}\right] \delta \xi+Q\left[\begin{array}{cc}
G & 0 \\
0 & -I
\end{array}\right]\left[\begin{array}{c}
\delta v \\
\delta \psi
\end{array}\right]=0
$$

where

$$
Q=\left[\begin{array}{lr}
Q_{1} & 0 \\
0 & Q_{2}
\end{array}\right]
$$

and $Q_{1} \in \mathfrak{R}^{n \times n}$ and $Q_{2} \in \Re^{q \times q}$. The least squares estimate of $\delta \xi$ is then given by [3]

$$
\begin{aligned}
\delta \xi= & {\left[F^{T} Q_{1}^{T} Q_{1} F+H^{T} Q_{2}^{T} Q_{2} H\right]^{-1} } \\
& \times\left[-F^{T} Q_{1}^{T} Q_{1} G \quad H^{T} Q_{2}^{T} Q_{2}\right]\left[\begin{array}{c}
\delta v \\
\delta \psi
\end{array}\right] \\
= & M\left[\begin{array}{c}
\delta v \\
\delta \psi
\end{array}\right] .
\end{aligned}
$$

This gives a static estimate of $\delta \xi$, but as with the observer design, there are a large number of parameters for on-line implementation and calibration. Therefore, we analyze this static representation of the states to identify which inputs and outputs have the most influence on the estimate.

\section{Singular Value Decomposition}

Consider the turbocharged system defined by (1). We would like to construct a static estimate of $P_{e}$ from measured variables and known inputs. Based on our equilibrium analysis, we construct $M$ from (2), with $Q$ selected to emphasize the fast, accurate pressure measurements. The wastegate flow input is deemphasized via $Q$, since measurement or estimation of this quantity is difficult in practice,

$$
Q=\left[\begin{array}{cccccc}
1.0 & 0 & 0 & 0 & 0 & 0 \\
0 & 1.0 & 0 & 0 & 0 & 0 \\
0 & 0 & 0.01 & 0 & 0 & 0 \\
0 & 0 & 0 & 1.0 & 0 & 0 \\
0 & 0 & 0 & 0 & 100 & 0 \\
0 & 0 & 0 & 0 & 0 & 100
\end{array}\right]
$$

By analyzing the singular value decomposition (SVD) of $M$ we can identify a set of inputs and outputs that have significant influence on the estimate of $\delta x$, and specifically $\delta P_{e}$,

$$
\delta x=M\left[\begin{array}{l}
\delta u \\
\delta y
\end{array}\right]=U S V^{T}\left[\begin{array}{l}
\delta u \\
\delta y
\end{array}\right] .
$$

Therefore, since $U$ is orthonormal,

$$
U^{T} \delta x=S V^{T}\left[\begin{array}{l}
\delta u \\
\delta y
\end{array}\right]
$$

Examination of the rows of $U^{T}$ and $S V^{T}$ tell us how much information from $u$ and $y$ is transmitted to the linear combination of $x$. For example, consider the system (1) when the engine is operating at approximately $3000 \mathrm{RPM}$ and $70 \mathrm{Nm}$,

$$
\begin{gathered}
U^{T}=\left[\begin{array}{cccc}
-0.02 & 0.05 & 0.99 & -0.04 \\
-0.78 & -0.16 & -0.03 & -0.61 \\
-0.15 & 0.99 & -0.05 & -0.07 \\
-0.61 & -0.04 & 0.02 & 0.79
\end{array}\right] \\
S=\left[\begin{array}{cccccc}
7.22 & 0 & 0 & 0 & 0 & 0 \\
0 & 1.25 & 0 & 0 & 0 & 0 \\
0 & 0 & 0.92 & 0 & 0 & 0 \\
0 & 0 & 0 & 0.02 & 0
\end{array}\right] \\
V^{T}=\left[\begin{array}{cccccc}
0.89 & -0.06 & 0.02 & -0.13 & -0.18 & 0.39 \\
-0.11 & 0.01 & 0 & 0.03 & -0.97 & -0.20 \\
-0.41 & 0.03 & 0 & 0.06 & -0.13 & 0.90 \\
-0.16 & -0.41 & 0.10 & -0.89 & -0.01 & 0 \\
0 & -0.05 & 0.99 & 0.14 & 0 & 0 \\
0 & -0.91 & -0.10 & 0.40 & 0 & 0
\end{array}\right] .
\end{gathered}
$$

Examining the first row of (5), we see that

$$
\begin{gathered}
-0.02 \delta P_{i}+0.05 \delta P_{t i p}+0.99 \delta P_{e}-0.04 \delta N_{t c}= \\
6.43 \delta E T C-0.43 \delta V C T+0.14 \delta W_{w g}-0.94 \delta N \\
-1.30 \delta P_{t i p}+2.82 \delta P_{i} .
\end{gathered}
$$

Since the contributions of turbocharger speed and wastegate flow rate are small in a relative sense, we approximate $\delta P_{e}$ as

$$
\begin{array}{r}
\delta P_{e} \approx 2.84 \delta P_{i}-0.43 \delta V C T-0.94 \delta N \\
-1.35 \delta P_{t i p}+6.43 \delta E T C .
\end{array}
$$


This parameterization aligns well with our physical intuition that the pressure in the exhaust manifold varies with flow rate and restriction.

Specifically, we know from [6] that $P_{i}, N$ and $V C T$ can be used to represent cylinder air charge, and therefore air mass flow rate through the system. In addition, we know that flow rate through the turbine is only weakly dependent on turbine speed so that the effective size of the flow restriction due to the turbine is approximately constant. So we surmise that the first three parameters in equation (6) represent the effects of air flow rate due to the restriction imposed by the turbine.

This implies that the remaining variables, ETC and $P_{t i p}$, can be used to characterize the effects of the variable flow restriction due to the wastegate. To relate this to physical behavior, consider a steady state operating point defined by air flow rate. At each $P_{t i p}$ there is a unique value of ETC that delivers this desired air flow rate. Similarly, there is a unique wastegate position that delivers any given $P_{t i p}$ at this air flow rate. So at steady state, knowledge of $E T C$ and $P_{t i p}$ uniquely defines wastegate position.

Thus using our understanding of the physical behavior of the system and the variables identified through SVD analysis, we conclude that $W_{e x h}, P_{t i p}$ and $E T C$ can be used to parameterize the equilibrium values of $P_{e}$.

\section{Static Estimate}

We have identified a parameterization of exhaust manifold pressure through system inputs and outputs. The Implicit Function Theorem [5], applied in steady state to the system model (1), guarantees existence of a functional relationship between these parameters and $P_{e}$. A practical way of identifying the relationship in a production environment, where engine dynamometer data is readily available, is through regression.

To generate the same type of data in our modeling environment, we simulate the nonlinear model of the turbocharged system (1) [2] over the possible range of $P_{t i p}$ and ETC combinations. These data are used to produce the least squares estimate given by

$$
\begin{aligned}
\hat{P}_{e}= & \alpha_{0}+\alpha_{1} P_{t i p}+\alpha_{2} E T C+\alpha_{3} W_{e x h} P_{t i p} \\
& +\alpha_{4} P_{t i p}^{2}+\alpha_{5} W_{e x h}^{2}
\end{aligned}
$$

where $\hat{P}_{e}$ is the estimate of absolute exhaust manifold pressure. The terms of (7) are chosen to achieve the best least squares fit.

This approach produces an accurate steady state estimate of the simulated exhaust manifold pressure, as demonstrated in Figure 3, which plots the estimated values of $P_{e}$ versus values generated with the full nonlinear model.

This estimator performs well at steady state but it is also important to consider the physical aspects of the dynamic environment. Transient simulation of the estimator reveals significant error when wastegate is changed quickly. This is demonstrated by example with the step responses shown by the dotted lines

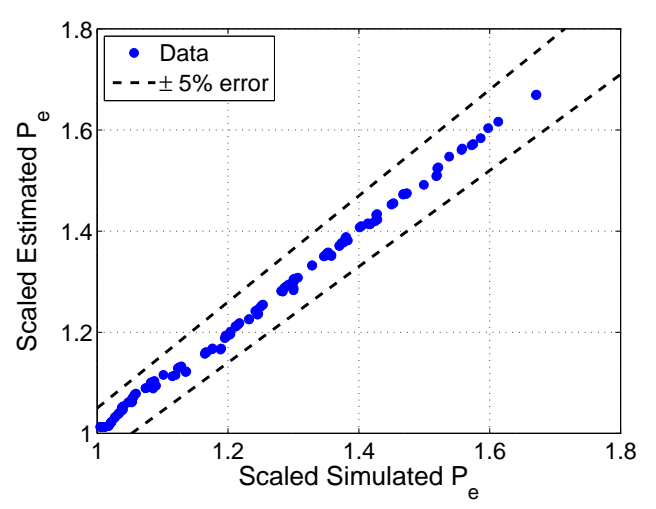

Figure 3. STEADY STATE PREDICTION OF $P_{e}$ USING THE STATIC ESTIMATE.
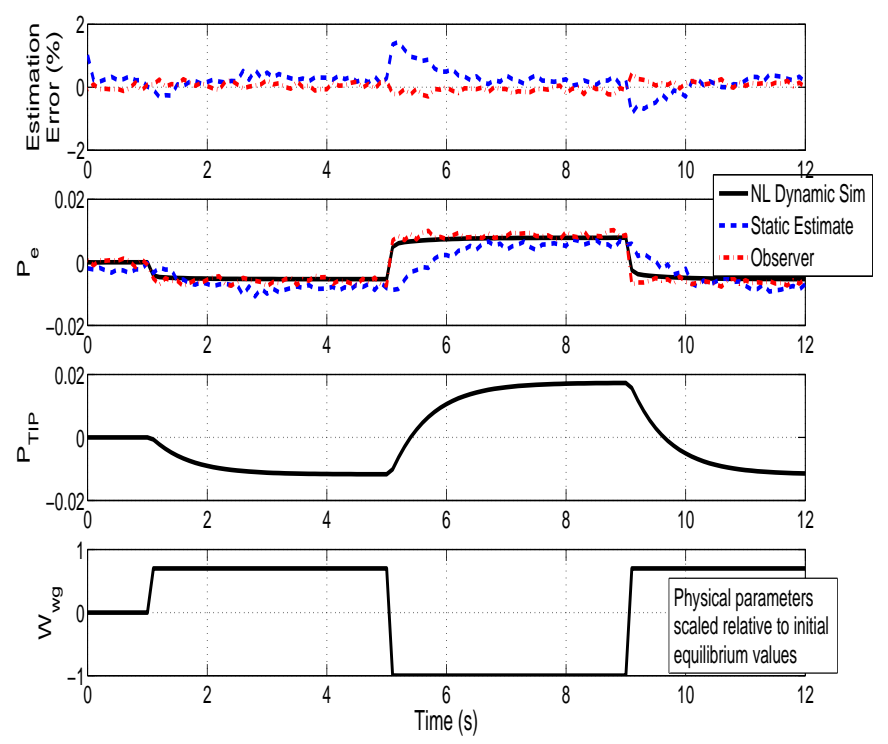

Figure 4. TRANSIENT PREDICTION OF $P_{e}$ USING THE STATIC ESTIMATE.

in Figure 4. Closer examination shows that $P_{t i p}$ responds relatively slowly to changes in wastegate, while $P_{e}$ initially responds very quickly. The relatively slow response of $P_{t i p}$ is due to the fact that the wastegate acts on $P_{t i p}$ via the turbocharger, which has a large inertia. The exhaust manifold, on the other hand, is small, with very fast dynamics and responds quickly to changes in wastegate. The estimate relying on $P_{t i p}$ is, therefore, unable to respond as quickly as desired.

\section{Static Estimate with Dynamic Compensation}

Transient analysis shows that our static estimate employing $P_{t i p}$ and $E T C$ is insufficient to capture exhaust manifold pressure dynamics. We now explore lead compensation as a means to improve the transient response of the estimator.

For ease of implementation, we consider a simple first order 

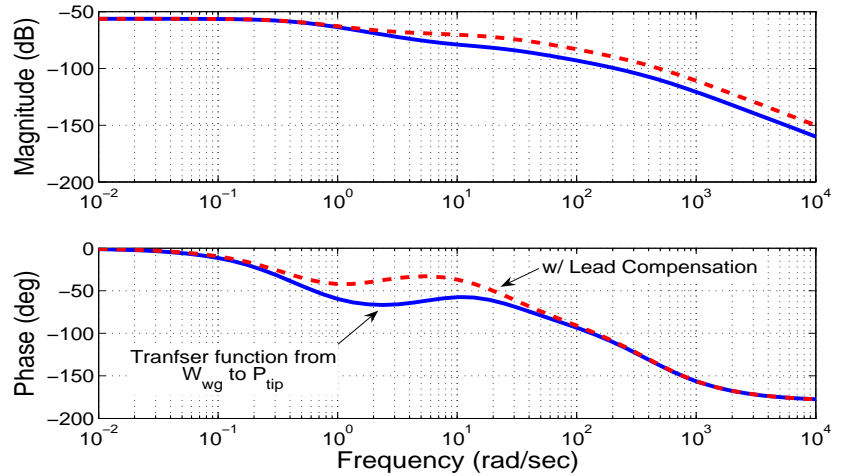

Figure 5. BODE DIAGRAM USED FOR LEAD COMPENSATION DEVELOPMENT.
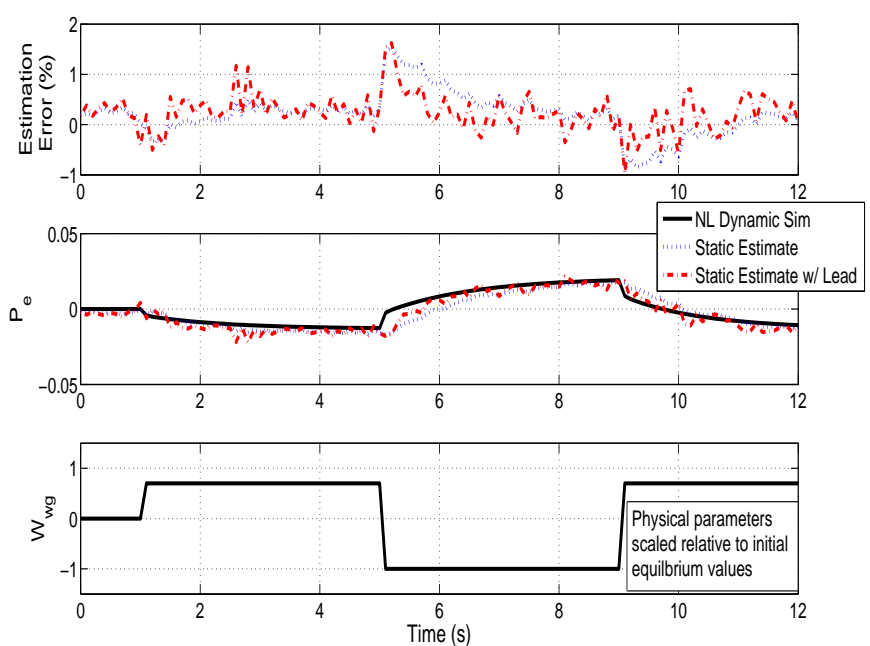

Figure 6. TRANSIENT PREDICTION OF $P_{e}$ USING THE LEAD COMPENSATED STATIC ESTIMATE.

filter that can be applied to the output of (7). Since it is the delay in response of $P_{t i p}$ to wastegate that is problematic for the static estimate, we examine the transfer function from $W_{w g}$ to $P_{t i p}$. The bode plot of this SISO system is shown in Figure 5. As a starting point, we choose a lead filter to extend the bandwidth of this system and then refine our design using nonlinear simulation of the MIMO system.

Nonlinear simulation quickly shows that the separation of the pole and zero of the lead filter is limited to approximately $\frac{1}{2}$ decade to prevent large overshoot and the associated increase in estimation error. With this constraint in mind, the zero location of 2.0 and the pole location of 6.25 are subjectively chosen by examining several nonlinear step responses. A small improvement in response for a step in wastegate command is shown in Figure 6. As expected with lead compensation, the effect of measurement noise is amplified thus reducing the overall benefit of the compensator.

Our compensator design is based on the characteristics of
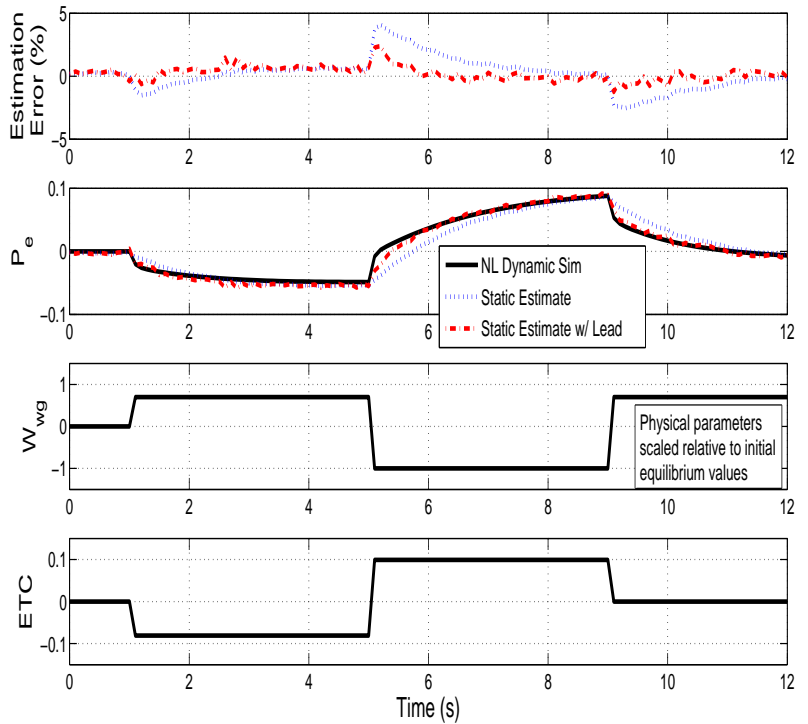

Figure 7. PREDICTION OF $P_{e}$ USING THE LEAD COMPENSATED STATIC ESTIMATE DURING A TRANSIENT DUE TO SIMULTANEOUS THROTTLE AND WASTEGATE COMMANDS.

the response of $P_{t i p}$ to wastegate. The throttle, however, also significantly influences $P_{t i p}$. Initially, $P_{t i p}$ can actually respond very quickly to a throttle command due to the abrupt change in flow rate leaving the volume. Therefore, we need to consider estimator performance in the presence of a throttle input as well. The system response to simultaneous inputs of throttle and wastegate is shown in Figure 7. Here we see no adverse effects due to throttle and in fact there is a substantial reduction in estimation error with lead compensation.

These results suggest that this simple approach may provide a practical strategy for estimating exhaust manifold pressure. Further analysis is required, however, to fully assess robustness to measurement noise and to determine if the filter coefficients must be scheduled with operating condition.

\section{CONCLUSIONS AND FUTURE WORK}

Model-based analysis has been used to assess the feasibility of estimating exhaust manifold pressure for a turbocharged gasoline engine with an algorithm that is computationally efficient and simple to calibrate. A traditional reduced order linear observer was developed to show that an estimator of this type is indeed feasible. The observer, however, has far too many calibration parameters for practical implementation. Therefore, equilibrium analysis was used to find a parameterization of $P_{e}$ through the inputs and outputs of the system. A static estimator based on $W_{\text {exh }}, P_{\text {tip }}$ and ETC performed well in steady state but was found to be inadequate during transients. Lead compensation improved transient performance despite increased sensitivity to measurement noise. 
Further work will include a robustness and calibration assessment of lead compensation over the operating range of engine and if necessary, investigation of more sophisticated dynamic compensators for the static estimator. Finally, the results will be validated with engine dynamometer testing.

\section{ACKNOWLEDGMENT}

The authors gratefully acknowledge Jeff Cook from the University of Michigan and Dave Hagner from Ford Motor Company for many valuable discussions and suggestions.

\section{REFERENCES}

[1] Per Andersson and Lars Eriksson, 2004. "Cylinder Air Charge Estimator in Turbocharged SI-Engines". Proceedings of the Society of Automotive Engineers World Congress. SAE-2004-01-1366.

[2] A. Karnik, J. Buckland and J. Freudenberg, 2005. "Electronic throttle and wastegate control for turbocharged gasoline engines". Proceedings of the American Control Conference, pt 7, Vol. 7, pp. 4434-9.

[3] John S. Bay, 1999. Fundamentals of Linear State Space Systems. McGraw-Hill, Inc.

[4] D.A. Lawrence and W.J. Rugh, 1995. "Gain Scheduling Linear Dynamic Controllers for a Nonlinear Plant". Automatica, 31(3):381-390

[5] N.B. Haaser and J.A. Sullivan, 1991. Real Analysis Dover Publication Inc., New York.

[6] M. Jankovic and S. Magner, 2002. "Variable Cam Timing: consequences to automotive engine control design. Proceedings of the $15^{\text {th }}$ Triennial World Congress of the International Federation of Automatic Control. 